\title{
Maps from Riemannian manifolds into non-degenerate Euclidean cones
}

\section{Luciano Mari and Marco Rigoli}

\begin{abstract}
Let $M$ be a connected, non-compact $m$-dimensional Riemannian manifold. In this paper we consider smooth maps $\varphi: M \rightarrow \mathbb{R}^{n}$ with images inside a non-degenerate cone. Under quite general assumptions on $M$, we provide a lower bound for the width of the cone in terms of the energy and the tension of $\varphi$ and a metric parameter. As a side product, we recover some well known results concerning harmonic maps, minimal immersions and Kähler submanifolds. In case $\varphi$ is an isometric immersion, we also show that, if $M$ is sufficiently well-behaved and has non-positive sectional curvature, $\varphi(M)$ cannot be contained into a non-degenerate cone of $\mathbb{R}^{2 m-1}$.
\end{abstract}

\section{Introduction}

In a famous paper Omori, [12], studied minimal immersions of manifolds into cones of Euclidean space and in the process he proved (a version of) what is by now known as the Omori-Yau maximum principle. This powerful tool has since then been applied to solve several geometric problems from Yau's pioneering work on the generalized Schwarz lemma, to the study of the group of conformal diffeomorphisms of a manifold, [14], to the theory of submanifolds (see also the very recent papers [3] and [2]) and so on; for a short account one can consult [13] and the references therein. The OmoriYau principle can be briefly stated in the following form:

Let $(M,\langle\rangle$,$) be a complete Riemannian manifold and let u \in$ $C^{2}(M)$ be a function satisfying $u^{*}=\sup _{M} u<+\infty$. Then,

2000 Mathematics Subject Classification: Primary 53C42, 35B50; Secondary 53C21. Keywords: Maximum principles, harmonic maps, isometric immersion, Riemannian manifold. 
under appropriate geometric assumptions on the manifold there exists a sequence $\left\{x_{k}\right\} \subset M$ with the following properties:
(i) $u\left(x_{k}\right)>u^{*}-\frac{1}{k}$,
(ii) $|\nabla u|\left(x_{k}\right)<\frac{1}{k}$
(iii) $\Delta u\left(x_{k}\right)<\frac{1}{k}$

for every $k \in \mathbb{N}$.

It turns out that in many applications property $(i i)$ plays no role. This fact suggests to ignore it and to look for possibly lighter geometric assumptions to ensure the validity of conclusions $(i)$ and (iii). Indeed, in [14] the authors show that the sole assumption of stochastic completeness of $(M,\langle$,$\rangle ) (that$ is, the property of the Brownian motion on $M$ to have infinite intrinsic lifetime) is equivalent to the simultaneous validity of $(i)$ and (iii) for every $u \in C^{2}(M), u^{*}<+\infty$, on an appropriate sequence.

To distinguish between the two situations we shall refer to this new statement as to the weak maximum principle. These two principles are indeed different. For instance, one immediately realizes that stochastic completeness does not even imply geodesic completeness (as an example, consider the standard punctured Euclidean space $\left.\mathbb{R}^{n} \backslash\{o\}\right)$.

As a matter of fact, geometric applications often lead to consider differential inequalities of the form

$$
\Delta u \geq b(x) f(u)
$$

for some $b(x) \in C^{0}(M), f(t) \in C^{0}(\mathbb{R})$. This and a number of other considerations have suggested to generalize the known results on stochastic completeness and the weak maximum principle to a wider class of elliptic operators. In particular, to symmetric diffusion operators $L=b^{-1} \operatorname{div}(A \nabla)$ on $L^{2}(M, b d x)$, where $A, b$ are positive smooth functions on $M$. Theorem 3.10 of [13] states the equivalence between L-stochastic completeness (here $L$ means that the diffusion having infinite intrinsic lifetime is that generated by $L$ ) and the weak maximum principle for $L$. For a detailed account, one can consult [13], [18], where the authors deal even with the case $u^{*}=+\infty$.

Together with diffusions, one can also consider the full Hessian operator. In this case we state the following definition:

The weak maximum principle for the Hessian operator holds on $M$ if for every $u \in C^{2}(M), u^{*}<+\infty$, there exists a sequence $\left\{x_{k}\right\} \subset M$ such that

$$
u\left(x_{k}\right)>u^{*}-\frac{1}{k}, \sup _{\substack{v \in T_{x_{k}} M \\|v|=1}} \operatorname{Hess}_{x_{k}} u(v, v)<\frac{1}{k} .
$$


It is worth to observe, in view of applications, that $L$-stochastic completeness and the weak maximum principle for the Hessian can be granted via the existence of appropriate proper functions defined in a neighborhood of infinity in $M$. This is achieved by the use of Khas'minskii test (see [13], where for convenience the result is stated for the Laplacian only) and of its generalization in the case of the Hessian (see again [13] and also question 33 in [16]). Observe that adding the condition $\left|\nabla u\left(x_{k}\right)\right|<1 / k$ to (1.1) gives the well known Omori-Yau maximum principle for the Hessian.

In this paper we shall make use of Theorem 1.9 (and subsequent remarks), Theorem 3.10 and Proposition 3.18 of [13] to prove the geometrical results that we are now going to describe.

Let $\left(\mathbb{R}^{n},\langle\rangle,\right), n \geq 2$, be the $n$-dimensional Euclidean space endowed with its canonical flat metric. Fix an origin $o \in \mathbb{R}^{n}$ and a unit vector $v \in \mathbb{S}^{n-1}$. We set $\mathcal{C}_{o, v, \theta}$, shortly $\mathcal{C}$, to denote the non-degenerate cone with vertex in $o$, direction $v$ and width $\theta, \theta \in(0, \pi / 2)$, that is,

$$
\mathcal{C}=\left\{z \in \mathbb{R}^{n} \backslash\{o\}:\left\langle\frac{z-o}{|z-o|}, v\right\rangle \geq \cos (\theta)\right\}
$$

Let $(M,()$,$) be a connected, m$-dimensional Riemannian manifold $(m \geq 2)$, and let

$$
\varphi:(M,(,)) \longrightarrow\left(\mathbb{R}^{n},\langle,\rangle\right)
$$

be a smooth map. We indicate with $|d \varphi|^{2}$ the square of the Hilbert-Schmidt norm of the differential $d \varphi$ (in other words, twice the energy density of $\varphi$ ) and with $\tau(\varphi)$ the tension field of $\varphi$. In case $\varphi$ is an isometric immersion, $|d \varphi|^{2}=m$ and $\tau(\varphi)=m H$, where $H$ is the mean curvature vector. We fix an origin $q \in M$ and we consider the distance function from $q, r(x)=d(x, q)$. We set $B_{r}$ for the geodesic ball with radius $r$ centered at $q$.

Since the work of Omori, the study of maps with image contained in a cone has captured the attention of researchers. Indeed, important progresses in the understanding of their geometry have been made by a number of authors and in particular, regarding the content of the present paper, by [4], [13] and by [1], where through stochastic methods it is proved that if $M$ is stochastically complete and $\varphi$ is an harmonic map with energy density bounded from below away from zero, $\varphi(M)$ cannot be contained into any non-degenerate cone of $\mathbb{R}^{n}$. However, all these references limit themselves to consider the harmonic (or minimal) case.

On the contrary, the two main results in this paper aim at determining a lower bound for the width of the cone containing $\varphi(M)$ when $\varphi$ is merely a smooth map or an isometric immersion, where of course we expect the estimate to depend on the position of $\varphi(M)$ in the cone. 
To state the next theorems, given $\eta>0$, we define

$$
A_{\eta}=\sup _{\substack{\xi \in(0,1) \\ \alpha \in(0, \min \{1, \eta \sqrt{1-\xi}\})}} \xi \alpha^{2} \sqrt{1-\alpha^{2}} .
$$

The constant $A_{\eta}$ can be easily computed, but the actual value is irrelevant for our purposes. Note also that $A_{\eta}$ is non-decreasing as a function of $\eta$.

Theorem 1.4. Let $M$ be a connected, non-compact m-dimensional Riemannian manifold, and let

$$
\varphi:(M,(,)) \longrightarrow\left(\mathbb{R}^{n},\langle,\rangle\right)
$$

be a map of class $C^{2}$ such that $|d \varphi(x)|^{2}>0$ on $M$. Consider the elliptic operator $L=|d \varphi|^{-2} \Delta$, and assume that $M$ is L-stochastically complete. Let $\mathcal{C}=\mathcal{C}_{o, v, \theta}$ be a cone with vertex at $o \in \mathbb{R}^{n} \backslash \varphi(M)$, let $\pi_{v}$ be the hyperplane orthogonal to $v$ passing through o and let $d\left(\pi_{v}, \varphi(M)\right)$ be the Euclidean distance between this hyperplane and $\varphi(M)$.

If $\varphi(M)$ is contained in $\mathcal{C}$, then

$$
\cos (\theta) \leq \sqrt{\frac{1}{A_{1}} d\left(\pi_{v}, \varphi(M)\right) \sup _{M}\left[\frac{|\tau(\varphi)|}{|d \varphi|^{2}}\right]}
$$

In case $\varphi$ is an isometric immersion, we can replace $A_{1}$ with $A_{m}$ in (1.5) obtaining a sharper estimate.

Remark 1. Note that, in case

$$
\sup _{M}\left[\frac{|\tau(\varphi)|}{|d \varphi|^{2}}\right]=+\infty
$$

and $d\left(\pi_{v}, \varphi(M)\right)=0$, that is, $\varphi(M)$ "gathers around the origin $o$ " as we shall see in the proof, we have no restriction on $\theta$.

Remark 2. The condition that $L=|d \varphi|^{-2} \Delta$ generates a conservative diffusion (i.e. $L$-stochastic completeness) is implied by

$$
\int_{0}^{\xi}|d \varphi|^{2} d t=+\infty \quad \mathbb{P}_{x} \text { a.s for some (hence, every) } x \in M,
$$

where the integral is evaluated along the paths of the Brownian motion $X_{t}$ starting from $x$, and $\xi$ is the intrinsic lifetime of $X_{t}$. As in the case of $\Delta$-stochastic completeness, no geodesic completeness is required. If $M$ is complete, $L$-stochastic completeness can be obtained using generalized 
versions of a previous work of Grigor'Yan [8], see [19] and [13] (Theorem 3.15 and subsequent propositions). In particular, if there exist $C>0, \beta \in \mathbb{R}$ such that

$$
|d \varphi(x)|^{2} \geq \frac{C}{(1+r(x))^{\beta}} \quad \text { on } M
$$

and

$$
\frac{r^{1-\beta}}{\log \left(\operatorname{Vol}\left(B_{r}\right)\right)} \notin L^{1}(+\infty)
$$

then the weak maximum principle for $L=|d \varphi|^{-2} \Delta$ holds. It is worth to observe that (1.7) implies $\beta \leq 2$, but no restriction on nonnegativity of $\beta$ is needed. In case $\beta=2$, an application of [19] leads to slightly improving (1.7) to

$$
\frac{\log r}{r \log \left(\operatorname{Vol}\left(B_{r}\right)\right)} \notin L^{1}(+\infty) .
$$

The converse of the above result is in general false; indeed, it is well known that, on a radially symmetric model in the sense of Greene and $\mathrm{Wu},[9]$, $\Delta$-stochastic completeness is equivalent to the condition

$$
\frac{\operatorname{Vol}\left(B_{r}\right)}{\operatorname{Vol}\left(\partial B_{r}\right)} \notin L^{1}(+\infty)
$$

which is implied by (1.7) with $\beta=0$ but not equivalent. We remark, in passing, that it is an open problem to establish whether or not (1.8) is sufficient for $\Delta$-stochastic completeness on a generic complete manifold.

Remark 3. (Sharpness of inequality 1.5) Due to the form of (1.5), we cannot expect the result to be significant when $\varphi(M)$ is far from $\pi_{v}$, in the following sense: for every $M, \mathcal{C}$ and $\varphi$ satisfying the assumptions of Theorem 1.4, and for every $k \geq 0$, we can consider the map $\varphi_{k}=\varphi+k v$. Then $d\left(\pi_{v}, \varphi_{k}(M)\right)=d\left(\pi_{v}, \varphi(M)\right)+k$, while the other parameters in the RHS of (1.5) remain unchanged. Therefore, for $k$ sufficiently large inequality (1.5) becomes meaningless unless $\tau(\varphi) \equiv 0$. On the contrary, we show with a simple example that, when $d\left(\pi_{v}, \varphi(M)\right)$ is very small, (1.5) is sharp in the following sense: for every fixed hyperplane $\pi_{v}$, and for every origin $o \in \pi_{v}$, there exists a family of maps $\varphi_{d}, d>0$ representing $d\left(\pi_{v}, \varphi_{d}(M)\right)$, such that, if we denote with $\theta_{d}$ the width of the non-degenerate tangent cone containing $\varphi_{d}(M)$,

$$
\frac{\cos ^{2}\left(\theta_{d}\right)}{d} \geq C \quad \text { when } \quad d \rightarrow 0^{+},
$$

for some constant $C>0$. Indeed, for every fixed $d>0$ consider the hypersurface $\varphi_{d}: \mathbb{R}^{m} \rightarrow \mathbb{R}^{m+1}$ given by the graph $\varphi(x)=\left(x,|x|^{2}+d\right)$, with the 
induced metric. Indicating with $\pi_{v}$ the hyperplane $x_{m+1}=0$, we have by standard calculations

$$
\left|\tau\left(\varphi_{d}\right)\right|=\frac{2 m+8(m-1)|x|^{2}}{\left(1+4|x|^{2}\right)^{3 / 2}} \quad \text { and } \quad\left|d \varphi_{d}\right|^{2}=m
$$

Therefore $\sup _{M}\left|\tau\left(\varphi_{d}\right)\right| /\left|d \varphi_{d}\right|^{2}=2$. Moreover, for the tangent cone passing through the origin

$$
\cos ^{2}\left(\theta_{d}\right)=\frac{4 d}{1+4 d}
$$

thus, since $d \equiv d\left(\pi_{v}, \varphi_{d}(M)\right)$, we reach the desired conclusion.

As an immediate consequence of Theorem 1.4, we recover Atsuji's result [1]:

Corollary 1.9. Let $\varphi: M \rightarrow \mathbb{R}^{n}$ be harmonic and such that $|d \varphi|^{2} \geq C$ for some positive constant $C$. If $M$ is stochastically complete, then $\varphi(M)$ cannot be contained in any non-degenerate cone of $\mathbb{R}^{n}$. In particular, a stochastically complete manifold cannot be minimally immersed into a nondegenerate cone of $\mathbb{R}^{n}$.

Note that even the statement of [1] in its full generality requires $|d \varphi|^{2} \geq$ $C>0$, an assumption that can be overcome by the weighted requests (1.6) and (1.7). Furthermore, in case $\beta=0$ we can replace $\Delta$-stochastic completeness and the uniform control from below in (1.6) with the properness of $\varphi$.

Corollary 1.10. Let $(M,()$,$) be a Riemannian manifold. Then, there does$ not exist any proper harmonic map $\varphi: M \rightarrow \mathbb{R}^{n}$, such that $|d \varphi(x)|>0$ on $M$ and $\varphi(M)$ is contained into a non-degenerate cone of $\mathbb{R}^{n}$.

Remark 4. It is a well known open problem to deal with the case $\theta=\pi / 2$, that is, when the cone degenerates to a half-space and the dimension $m$ is greater than 2. When $m=2, n=3$, by Hoffman-Meeks' half-space Theorem [10] the only properly embedded minimal surfaces in a half-space are affine planes. On the contrary, if $m \geq 3$ there exist properly embedded minimal hypersurfaces even contained between two parallel hyperplanes (the so called generalized catenoids). It is still an open problem to find sufficient conditions on $M, \varphi$ in order to have a Hoffman-Meeks' type result, and it seems quite difficult to adapt the methods of the proof of (1.4) for this purpose.

The next application of Theorem 1.4 has a topological flavor. This result, which is interesting when $\varphi$ is not proper, ensures that some kind of "patological" gathering around points of $\overline{\varphi(M)} \backslash \varphi(M)$ does not occur when the 
map is sufficiently well behaved. To make the corollary more transparent, we state it using the sufficient conditions (1.6) and (1.7). First we introduce the following

Definition 1.11. Let $\mathcal{S}$ be a convex subset of $\mathbb{R}^{n}$. A point $p \in \overline{\mathcal{S}}$ is called an $n$-corner of $\mathcal{S}$ if it is the vertex of a non-degenerate cone containing $\mathcal{S}$.

Corollary 1.12. Let $(M,()$,$) be a complete Riemannian manifold and let$ $\varphi: M \rightarrow \mathbb{R}^{n}$ be a map of class $C^{2}$. Suppose that (1.6) holds, and that

$$
|\tau(\varphi)(x)| \leq \frac{\widetilde{C}}{r(x)^{\beta}} \quad \text { for } r(x) \gg 1
$$

for some $\widetilde{C}>0$ and $\beta \in \mathbb{R}$ as in (1.6). Assume also that (1.7) holds. Then, the convex envelope $\operatorname{Conv}(\varphi(M))$ contains no $n$-corners.

The second main theorem of this paper is obtained with a modification of the idea used in the proof of Theorem 1.4. We obtain

Theorem 1.14. Let $\varphi:(M,(),) \rightarrow \mathbb{R}^{n}$ be an isometric immersion of an $m$ dimensional manifold satisfying the weak maximum principle for the Hessian into a non-degenerate cone $\mathcal{C}_{o, v, \theta}$. Assume the codimension restriction

$$
0<n-m<m
$$

and suppose that the sectional curvature of $M$ satisfy

$$
{ }^{M} \operatorname{Sect}_{x} \leq \chi^{2} \quad \forall x \in M
$$

for some constant $\chi \geq 0$. Then

$$
\cos (\theta) \leq \sqrt{d\left(\pi_{v}, \varphi(M)\right) \frac{\chi}{A_{1}}}
$$

where $A_{1}$ is as in (1.3).

Remark 5. With the notation ${ }^{M}$ Sect $_{x} \leq g(x)$ for some function $g$ on $M$, we mean that, for every $x \in M$ and every 2-plane $\pi \leq T_{x} M$, the sectional curvature of $\pi$ in $x$ satisfy ${ }^{M} \operatorname{Sect}_{x}(\pi) \leq g(x)$.

As a consequence, we get the following corollaries: the former generalizes results of Tompkins [20], Chern-Kuiper [6] and Jorge-Koutroufiotis [11], whereas the latter improves on [7, Theorem 8.3]. 
Corollary 1.18. Let $(M,\langle\rangle$,$) be a complete m-dimensional Riemannian$ manifold with sectional curvature satisfying

$$
-B^{2}\left(1+r(x)^{2}\right) \prod_{j=1}^{N} \log ^{(j)} r(x) \leq{ }^{M} \text { Sect }_{x} \leq 0,
$$

for some $B>0, N \in \mathbb{N}$ and where $\log ^{(j)}$ stands for the $j$-iterated logarithm. Then, $M$ cannot be isometrically immersed into a non-degenerate cone of $\mathbb{R}^{2 m-1}$.

Corollary 1.20. Let $\left(M^{2 m},\langle\rangle, J,\right)$ be a Kähler manifold such that the weak maximum principle for the Hessian holds. Then $\varphi(M)$ cannot be isometrically immersed into a non-degenerate cone of $\mathbb{R}^{3 m-1}$.

Remark 6. The inequality on the left of (1.19) provides a sharp sufficient condition for the full Omori-Yau maximum principle for the Hessian. Indeed, it would be sufficient that this inequality hold along 2-planes containing $\nabla r$ (see [13, Theorem 1.9]). As far as we know, it is an open problem to obtain other general sufficient conditions ensuring the validity of the weak maximum principle for the Hessian.

\section{Proof of the Theorems}

\subsection{Proof of Theorem 1.4}

First of all we observe that

$$
d\left(\pi_{v}, \varphi(M)\right)=\inf _{x_{o} \in M}\left\langle\varphi\left(x_{o}\right), v\right\rangle
$$

and that the right hand side of (1.5) is invariant under homothetic transformations of $\mathbb{R}^{n}$. We choose $o$ as the origin of global coordinates, and for the ease of notation we set

$$
b=\cos (\theta) \quad b \in(0,1) .
$$

Furthermore, for future use, note that $\varphi(M) \subseteq \mathcal{C}$ implies

$$
\langle\varphi(x), v\rangle \geq b|\varphi(x)|>0 \quad \forall x \in M .
$$

Next, we reason by contradiction and we suppose that (1.5) is false. Therefore, there exists $x_{o} \in M$ such that

$$
\left\langle\varphi\left(x_{o}\right), v\right\rangle \sup _{x \in M}\left[\frac{|\tau(\varphi(x))|}{|d \varphi(x)|^{2}}\right]<A_{1} b^{2} .
$$


By definition, and the fact that the inequality is strict, we can find

$$
\xi \in(0,1), \quad \alpha \in(0, \sqrt{(1-\xi)})
$$

such that

$$
\left\langle\varphi\left(x_{o}\right), v\right\rangle \sup _{x \in M}\left[\frac{|\tau(\varphi(x))|}{|d \varphi(x)|^{2}}\right]<\left(\xi \alpha^{2} \sqrt{1-\alpha^{2}}\right) b^{2},
$$

thus:

$$
\left\langle\varphi\left(x_{o}\right), v\right\rangle|\tau(\varphi(x))|<\left(\xi \alpha^{2} \sqrt{1-\alpha^{2}}\right) b^{2}|d \varphi(x)|^{2} \quad \forall x \in M .
$$

For the ease of notation we set $T=\left\langle\varphi\left(x_{o}\right), v\right\rangle>0$ and $a=b \alpha$; the last relation becomes

$$
T|\tau(\varphi(x))|<\frac{\xi a^{2} \sqrt{b^{2}-a^{2}}}{b}|d \varphi(x)|^{2} \quad \forall x \in M .
$$

Note also that

$$
a \in(0, b \sqrt{(1-\xi)}) \subseteq(0, b)
$$

Now, we define the following function:

$$
u(x)=\sqrt{T^{2}+a^{2}|\varphi(x)|^{2}}-\langle\varphi(x), v\rangle,
$$

and we note that, by construction, $u\left(x_{o}\right)>0$. We first claim that

$$
u<T \quad \text { on } M
$$

indeed, an algebraic manipulation shows that (2.5) is equivalent to

$$
\langle\varphi(x), v\rangle^{2}+2 T\langle\varphi(x), v\rangle-a^{2}|\varphi(x)|^{2}>0 \quad \text { on } M .
$$

On the other hand, using (2.1) the LHS of the above inequality is bounded from below by $\left(b^{2}-a^{2}\right)|\varphi(x)|^{2}>0$ since $a<b$, and the claim is proved.

We now consider the closed non empty set:

$$
\Omega_{o}=\left\{x \in M: u(x) \geq u\left(x_{o}\right)\right\} .
$$

Using (2.1) and the definition of $\Omega_{o}$ we deduce:

$$
\sqrt{T^{2}+a^{2}|\varphi(x)|^{2}} \geq b|\varphi(x)|+u\left(x_{o}\right) .
$$

Since $u\left(x_{o}\right)>0$ by construction, we can square inequality (2.6) to obtain

$$
\left(b^{2}-a^{2}\right)|\varphi(x)|^{2}+2 b u\left(x_{o}\right)|\varphi(x)|+u\left(x_{o}\right)^{2}-T^{2} \leq 0 .
$$


Since $\left(b^{2}-a^{2}\right)>0$, the LHS of the above inequality is a quadratic polynomial in $|\varphi(x)|$ with two distinct roots $\alpha_{-}<0<\alpha_{+}$(use Cartesio rule and (2.5)), where $\alpha_{ \pm}$are given by

$$
\alpha_{ \pm}=\left[b^{2}-a^{2}\right]^{-1}\left\{ \pm \sqrt{\left(b^{2}-a^{2}\right) T^{2}+a^{2} u\left(x_{o}\right)^{2}}-b u\left(x_{o}\right)\right\}
$$

therefore, (2.7) implies

$$
|\varphi(x)| \leq\left[b^{2}-a^{2}\right]^{-1}\left\{\sqrt{\left(b^{2}-a^{2}\right) T^{2}+a^{2} u\left(x_{o}\right)^{2}}-b u\left(x_{o}\right)\right\} \quad \text { on } \Omega_{o} .
$$

We then use the elementary inequality $\sqrt{1+t^{2}} \leq 1+t$ on $\mathbb{R}_{0}^{+}$to deduce

$$
\begin{aligned}
{\left[b^{2}-a^{2}\right]^{-1}\left\{\sqrt{\left(b^{2}-a^{2}\right) T^{2}+a^{2} u\left(x_{o}\right)^{2}}-b u\left(x_{o}\right)\right\}=} \\
\quad=\frac{a u\left(x_{o}\right)}{b^{2}-a^{2}} \sqrt{1+\frac{\left(b^{2}-a^{2}\right) T^{2}}{a^{2} u\left(x_{o}\right)^{2}}}-\frac{b u\left(x_{o}\right)}{b^{2}-a^{2}} \\
\leq \frac{a u\left(x_{o}\right)}{b^{2}-a^{2}}\left(1+\frac{T \sqrt{b^{2}-a^{2}}}{a u\left(x_{o}\right)}\right)-\frac{b u\left(x_{o}\right)}{b^{2}-a^{2}} \\
\quad=\frac{T}{\sqrt{b^{2}-a^{2}}}-\frac{u\left(x_{o}\right)}{b+a}
\end{aligned}
$$

and thus (2.8) together with $u\left(x_{o}\right)>0$ yields

$$
|\varphi(x)| \leq \frac{T}{\sqrt{b^{2}-a^{2}}}=\varphi_{\max } \quad \text { on } \Omega_{o} .
$$

To compute $\Delta u$, we fix a local orthonormal frame $\left\{e_{i}\right\}$ and its dual coframe $\left\{\theta^{i}\right\}$. Then, writing $d u=u_{i} \theta^{i}$, a simple computation shows that

$$
u_{i}=\frac{a^{2}\left\langle d \varphi\left(e_{i}\right), \varphi\right\rangle}{\sqrt{T^{2}+a^{2}|\varphi|^{2}}}-\left\langle d \varphi\left(e_{i}\right), v\right\rangle,
$$

and taking the covariant derivative we have $\nabla d u=u_{i j} \theta^{i} \otimes \theta^{j}$, where

$$
\begin{aligned}
u_{i j}= & -\frac{a^{4}\left\langle d \varphi\left(e_{i}\right), \varphi\right\rangle\left\langle d \varphi\left(e_{j}\right), \varphi\right\rangle}{\left(T^{2}+a^{2}|\varphi|^{2}\right)^{3 / 2}}-\left\langle\nabla d \varphi\left(e_{i}, e_{j}\right), v\right\rangle \\
& +\frac{a^{2}\left\langle\nabla d \varphi\left(e_{i}, e_{j}\right), \varphi\right\rangle+a^{2}\left\langle d \varphi\left(e_{i}\right), d \varphi\left(e_{j}\right)\right\rangle}{\sqrt{T^{2}+a^{2}|\varphi|^{2}}} .
\end{aligned}
$$

Tracing the above expression we get

$$
\Delta u=\left\langle\frac{S}{|\varphi|} \varphi-v, \tau(\varphi)\right\rangle+S \frac{|d \varphi|^{2}}{|\varphi|}-\frac{1}{|\varphi|^{2}} \frac{S^{2}}{\sqrt{T^{2}+a^{2}|\varphi|^{2}}} \sum_{i=1}^{m}\left\langle\varphi, d \varphi\left(e_{i}\right)\right\rangle^{2}
$$


on $M$, where we have defined

$$
S=S(x)=\frac{a^{2}|\varphi(x)|}{\sqrt{T^{2}+a^{2}|\varphi(x)|^{2}}}
$$

Note that, by (2.1),

$$
\left|\frac{S}{|\varphi|} \varphi-v\right|^{2} \leq S^{2}-2 b S+1
$$

and that

$$
\sum_{i=1}^{m}\left\langle\varphi, d \varphi\left(e_{i}\right)\right\rangle^{2} \leq\left\{\begin{array}{l}
|\varphi|^{2} \sum_{i=1}^{m}\left|d \varphi\left(e_{i}\right)\right|^{2}=|\varphi|^{2}|d \varphi|^{2} \\
|\varphi|^{2}=\frac{1}{m}|\varphi|^{2}|d \varphi|^{2} \quad \text { if } \varphi \text { is isometric. }
\end{array}\right.
$$

The possibility, for the isometric case, of substituting $A_{1}$ with $A_{m}$ in (1.5) depends only on the above difference. Since the next passages are the same, we carry on with the general case. Substituting (2.13), (2.14) in (2.11) it follows that

$$
\Delta u \geq-|\tau(\varphi)| \sqrt{S^{2}-2 b S+1}+S \frac{|d \varphi|^{2}}{|\varphi|}-\frac{S^{2}}{\sqrt{T^{2}+a^{2}|\varphi|^{2}}}|d \varphi|^{2} .
$$

We now restrict our estimates on the RHS of (2.15) on $\Omega_{o}$. Then, (2.9) holds and from (2.3) we obtain

$$
\frac{|\tau(\varphi)|}{|d \varphi|^{2}}<\frac{\xi a^{2} \sqrt{b^{2}-a^{2}}}{T b}=\frac{\xi a^{2}}{\sqrt{T^{2}+a^{2} \varphi_{\max }^{2}}} \leq \frac{\xi a^{2}}{\sqrt{T^{2}+a^{2}|\varphi|^{2}}}=\frac{\xi S}{|\varphi|} .
$$

Inserting this inequality into (2.15) we have

$$
\Delta u \geq \frac{a^{2}|d \varphi|^{2}}{\sqrt{T^{2}+a^{2}|\varphi|^{2}}}\left[1-\xi \sqrt{S^{2}-2 b S+1}-\frac{S^{2}}{a^{2}}\right] .
$$

We want to find a strictly positive lower bound for $\left(1-\xi \sqrt{S^{2}-2 b S+1}-\right.$ $\left.S^{2} / a^{2}\right)$ on $\Omega_{o}$. Since $1-2 b S+S^{2}$ represents a convex parabola and since $S$ is increasing in the variable $|\varphi|$ on $\left[0, \varphi_{\max }\right]$, its maximum is attained either in 0 or in $\varphi_{\max }$. Since $S(0)=0, S\left(\varphi_{\max }\right)=a^{2} / b>0$ we have

$$
S\left(\varphi_{\max }\right)^{2}-2 b S\left(\varphi_{\max }\right)+1=1+a^{2}\left(\frac{a^{2}}{b^{2}}-2\right)<1=S(0)^{2}-2 b S(0)+1,
$$


thus we can roughly bound as follows:

$$
1-\xi \sqrt{S^{2}-2 b S+1}-\frac{S^{2}}{a^{2}} \geq 1-\xi-\frac{a^{2}}{b^{2}}
$$

and the RHS of the above inequality is strictly positive since $a \in(0, b \sqrt{1-\xi})$. Therefore, (2.16) together with (2.9) yield

$$
L u=|d \varphi|^{-2} \Delta u \geq \frac{a^{2}}{\sqrt{T^{2}+a^{2}|\varphi|^{2}}}\left[1-\xi-\frac{a^{2}}{b^{2}}\right] \geq \delta \quad \text { on } \Omega_{o},
$$

for some $\delta>0$.

There are now two possibilities:

i) $x_{o}$ is an absolute maximum for $u$ on $M$. By assumption $\left|d \varphi\left(x_{o}\right)\right|^{2}>0$, and the finite form of the maximum principle yields $\Delta u\left(x_{o}\right) \leq 0$, so that $L u\left(x_{o}\right) \leq 0$. Since $x_{o} \in \Omega_{o}(2.17)$ immediately gives the contradiction.

ii) $\operatorname{Int}\left(\Omega_{o}\right)=\left\{x \in M: u(x)>u\left(x_{o}\right)\right\} \neq \emptyset$. In this case, since $u(x)$ is bounded above on $M$, it is enough to evaluate inequality (2.17) along a sequence $\left\{x_{k}\right\}$ realizing the weak maximum principle for $L$, that is $u\left(x_{k}\right)>u^{*}-1 / k, L u\left(x_{k}\right)<1 / k$. Note that this sequence eventually lies in $\operatorname{Int}\left(\Omega_{o}\right)$.

This concludes the proof.

\subsection{Proof of Corollary 1.9}

If $M$ is stochastically complete and $|d \varphi|^{2} \geq C$, then it is straightforward to deduce that $M$ is $L$-stochastically complete, where $L=|d \varphi|^{-2} \Delta$. Indeed, for every $u \in C^{2}(M)$ with $u^{*}<+\infty$, along the sequence $\left\{x_{k}\right\}$ realizing the weak maximum principle for $\Delta$ we have also

$$
L u\left(x_{k}\right)=\left|d \varphi\left(x_{k}\right)\right|^{-2} \Delta u\left(x_{k}\right) \leq \frac{1}{C k} .
$$

The result follows setting $\tau(\varphi) \equiv 0$ in Theorem 1.4.

\subsection{Proof of Corollary 1.10}

From $(2.9)$ in the proof of Theorem 1.4 we deduce that $\varphi\left(\Omega_{o}\right)$ is bounded, hence $\overline{\varphi\left(\Omega_{0}\right)}$ is compact. The properness assumption implies that $\varphi^{-1}\left(\overline{\varphi\left(\Omega_{o}\right)}\right)$ is compact, thus $\Omega_{o}$ is compact. Therefore, it is enough to use the finite form of the maximum principle in (2.17). 


\subsection{Proof of Corollary 1.12}

We reason by contradiction and let $p \in \operatorname{Conv}(\varphi(M))$ be an $n$-corner.

- If $p \in \operatorname{Conv}(\varphi(M)) \backslash \overline{\varphi(M)}$ fix a small ball around $p$ contained in $\mathbb{R}^{n} \backslash \overline{\varphi(M)}$, and cut the corner transversally with an hyperplane sufficiently near to $p$; it is immediate to see that in this way we produce a convex set containing $\varphi(M)$ and strictly smaller than $\operatorname{Conv}(\varphi(M))$, contradiction.

- Suppose now $p \in \varphi(M)$, and let $x \in M$ such that $\varphi(x)=p$. Consider the map $d \varphi_{\mid x}$; by assumption, there exists a direction $v \in T_{x} M$ such that $\left|d \varphi_{\mid x} v\right| \neq 0$, thus by continuity we can take a curve

$$
\gamma:(-\varepsilon, \varepsilon) \rightarrow M \quad, \quad \gamma(0)=x \quad, \quad \gamma^{\prime}(0)=v
$$

with $\varepsilon$ small such that $\left|d \varphi_{\mid \gamma(t)}\left(\gamma^{\prime}(t)\right)\right| \neq 0$ on $(-\varepsilon, \varepsilon)$. Therefore, $\varphi \circ \gamma$ is an immersed curve in $\mathbb{R}^{n}$, and this fact contradicts the assumption that $p$ is an $n$-corner.

- If $p \in \overline{\varphi(M)} \backslash \varphi(M)$, choose $\pi_{v}$ as the hyperplane orthogonal to the direction of the cone and passing through $p$. It follows that $d\left(\varphi(M), \pi_{v}\right)$ $=0$. By (1.6) and (1.13), we argue that $|\tau(\varphi)| /|d \varphi|^{2}$ is bounded above on $M$. By Remark 2, (1.6) and (1.7) ensure that $M$ is $L$-stochastically complete, where $L=|d \varphi|^{-2} \Delta$. By Theorem 1.4 we conclude the validity of (1.5) which gives $\theta=\pi / 2$, contradiction.

\subsection{Proof of Theorem 1.14}

We follow the proof of Theorem 1.4 verbatim substituting (2.2) with

$$
\left\langle\varphi\left(x_{o}\right), v\right\rangle \chi<\left(\xi \alpha^{2} \sqrt{1-\alpha^{2}}\right) b^{2}
$$

for some $\xi \in(0,1), \alpha \in(0, \sqrt{1-\xi}),(2.3)$ with

$$
T \chi<\frac{\xi a^{2} \sqrt{b^{2}-a^{2}}}{b}
$$

and arrive up to inequality (2.9) included. Next, we fix $x \in \Omega_{o}$ and we let $X, Y \in T_{x} M$ be orthonormal vectors spanning the 2-plane $\pi$. From Gauss equations and (1.16) we have

$$
\langle I I(X, X), I I(Y, Y)\rangle-|I I(X, Y)|^{2}={ }^{M} \operatorname{Sect}(\pi) \leq \chi^{2},
$$

where $I I$ is the second fundamental form. 
Since $0<n-m<m$, by Otsuki lemma (see [7, Lemma 3.1]) it follows that there exists a unit vector $W \in T_{x} M$ such that

$$
|I I(W, W)| \leq \chi
$$

hence, from (2.19) and (2.9) we deduce

$$
|I I(W, W)|<\frac{\xi a^{2} \sqrt{b^{2}-a^{2}}}{T b}=\frac{\xi a^{2}}{\sqrt{T^{2}+a^{2} \varphi_{\max }^{2}}} \leq \frac{\xi a^{2}}{\sqrt{T^{2}+a^{2}|\varphi(x)|^{2}}} .
$$

Next, we let $\gamma:[0, \varepsilon) \rightarrow M, \varepsilon>0$, be the geodesic characterized by the initial data

$$
\gamma(0)=x \quad, \quad \gamma^{\prime}(0)=W .
$$

Call $s \in[0, \varepsilon)$ the arc-length parameter and define the function

$$
g:[0, \varepsilon) \rightarrow \mathbb{R} \quad g(s)=u(\gamma(s)) .
$$

A simple computation, using the fact that $\varphi$ is an isometric immersion, gives:

$$
\begin{aligned}
g^{\prime \prime}(s)= & \left\langle\frac{S}{|\varphi(\gamma)|} \varphi(\gamma)-v, I I\left(\gamma^{\prime}, \gamma^{\prime}\right)\right\rangle+\frac{S}{|\varphi(\gamma)|} \\
& -\frac{S^{3}}{a^{2}|\varphi(\gamma)|^{3}}\left\langle d \varphi\left(\gamma^{\prime}\right), \varphi(\gamma)\right\rangle^{2},
\end{aligned}
$$

where $S$ has the expression in (2.12), evaluated at $x=\gamma(s)$. Since

$$
\left|\frac{S}{|\varphi|} \varphi-v\right|^{2} \leq 1+S^{2}-2 b S, \quad\left\langle d \varphi\left(\gamma^{\prime}\right), \varphi\right\rangle^{2} \leq\left|d \varphi\left(\gamma^{\prime}\right)\right|^{2}|\varphi|^{2}=|\varphi|^{2},
$$

setting $S_{o}=S(\gamma(0))$, evaluating at $s=0$ we deduce

$$
g^{\prime \prime}(0) \geq-|I I(W, W)| \sqrt{S_{o}^{2}-2 b S_{o}+1}+\frac{a^{2}-S_{o}^{2}}{\sqrt{T^{2}+a^{2}|\varphi(\gamma)|^{2}}} .
$$

Inserting (2.21) into (2.23) we get

$$
g^{\prime \prime}(0) \geq \frac{a^{2}}{\sqrt{T^{2}+a^{2}|\varphi(\gamma)|^{2}}}\left[1-\xi \sqrt{1+S_{o}^{2}-2 b S_{o}}-\frac{S_{o}^{2}}{a^{2}}\right] .
$$

Proceeding as in the proof of Theorem 1.4, since $a \in(0, b \sqrt{1-\xi}) \subset(0, b)$

$$
g^{\prime \prime}(0) \geq \frac{a^{2}}{\sqrt{T^{2}+a^{2}|\varphi(\gamma)|^{2}}}\left[1-\xi-\frac{a^{2}}{b^{2}}\right] \geq \frac{a^{2} \sqrt{b^{2}-a^{2}}}{b T}\left[1-\xi-\frac{a^{2}}{b^{2}}\right]=\delta>0,
$$

where $\delta$ is independent from $x \in \Omega_{o}$ and from $W$. 
On the other hand, a standard computation using the fact that $\gamma$ is a geodesic and the definition of the Hessian of a function, gives $g^{\prime \prime}(0)=$ $\operatorname{Hess}_{x} u(W, W)$. Putting together the last two inequalities we obtain

$$
\operatorname{Hess}_{x} u(W, W) \geq \delta>0 .
$$

If $x_{o}$ is an absolute maximum of $u$, then from (2.25) we immediately contradict the finite maximum principle, otherwise

$$
\operatorname{Int}\left(\Omega_{o}\right)=\left\{x \in M: u(x)>u\left(x_{o}\right)\right\} \neq \emptyset
$$

and (2.25) gives

$$
\inf _{x \in \operatorname{Int}\left(\Omega_{o}\right)} \sup _{\substack{Y \in T_{x} M \\|Y|=1}} \operatorname{Hess}_{x} u(Y, Y) \geq \delta>0,
$$

contradicting the validity of the weak maximum principle for the Hessian operator since the function $u$ in bounded above on $M$. This completes the proof of Theorem 1.14.

\subsection{Proof of Corollary 1.18}

By (1.19), using Theorem 1.9 of [13] we have the validity of the weak maximum principle for the Hessian. The result follows immediately setting $\chi=0$ and $n=2 m-1$.

\subsection{Proof of Corollary 1.20}

The proof follows the same lines as in [7], so we only sketch it. From the assumptions, since the codimension is $m-1<m$, for every $x \in M$ the theory of flat bilinear forms ensure the existence of a vector $Z \in T_{x} M,|Z|=1$ such that $I I(J Z, J Z)=-I I(Z, Z)$. We define $u, \Omega_{o}$ as in Theorem 1.14. Expression (2.22) gives at every point $x$, and for every $X \in T_{x} M,|X|=1$

$$
\operatorname{Hess}_{x} u(X, X) \geq\left\langle\frac{S}{|\varphi(x)|} \varphi(x)-v, I I(X, X)\right\rangle+\frac{S}{|\varphi(x)|}\left(1-\frac{S^{2}}{a^{2}}\right) .
$$

This calculation is independent from the value of $a \in(0, b)$. If $a$ is chosen to be sufficiently small that $S^{2} / a^{2}<\delta<1$ (note that, by definition, on $\Omega_{o}$ it holds $S=O\left(a^{2}\right)$ and $\left.S /|\varphi| \geq a^{2} / T\right)$, evaluating along a sequence $\left\{x_{k}\right\}$ satisfying the weak maximum principle for the Hessian we deduce, for $k$ sufficiently large,

$$
\begin{aligned}
\left\langle\frac{S}{\left|\varphi\left(x_{k}\right)\right|} \varphi\left(x_{k}\right)-v, I I\left(X_{k}, X_{k}\right)\right\rangle & \leq \operatorname{Hess}_{x} u\left(X_{k}, X_{k}\right)-\frac{S}{\left|\varphi\left(x_{k}\right)\right|}(1-\delta) \\
& \leq \frac{1}{k}-\frac{a^{2}}{T}(1-\delta)<0
\end{aligned}
$$

for every $X_{k} \in T_{x_{k}} M,\left|X_{k}\right|=1$. This fact contradicts the existence of $Z$. 


\section{Ending remarks}

We end the paper with some observations regading Theorem 1.14 and Corollary 1.18. In particular, we stress the difference of applicability between the weak maximum principles for the Laplacian and for the full Hessian operator. A first striking difference is pointed out by Proposition 40 of [16], which states that every Riemannian manifold satisfying the weak maximum principle for the Hessian must be non-extendible (that is, non isometric to any proper open subset of another Riemannian manifold). For example, for every Riemannian manifold $M$ and $p \in M, M \backslash\{p\}$ does not satisfy the weak maximum principle for the Hessian. This is in sharp contrast to Theorem IX. 3 of [5], whose immediate application is the following

Proposition 3.1. Let $(M,\langle\rangle$,$) be a stochastically complete m$-dimensional manifold with Riemannian measure $\mu$, and let $K \subset M$ be a compact submanifold of dimension $k \geq 0$ such that $M \backslash K$ is connected. Then, if $m-k \geq 2$, $M \backslash K$ is stochastically complete.

Proof. Denoting with $B_{\varepsilon}=\{x: d(x, K)<\varepsilon\}$ and with $p_{\varepsilon}$ the Dirichlet heat kernel on $M \backslash B_{\varepsilon}$, by Theorem IX.1 of [5] $p_{\varepsilon} \uparrow p$ uniformly on compact subsets of $M \backslash K \times[0,+\infty)$. Therefore, the Dirichlet heat kernel $\widetilde{p}$ of $M \backslash K$ coincides with $p_{\mid M \backslash K}$, and since $\mu(K)=0$ the Brownian motion $X_{t}$ on $M \backslash K$ satisfies

$$
\mathbb{P}\left(X_{t} \in M \backslash K \mid X_{0}=x\right)=\int_{M \backslash K} \tilde{p}(x, y, t) d \mu(y)=\int_{M} p(x, y, t) d \mu(y)=1
$$

for every $t>0, x \in M \backslash K$, and this shows that $M \backslash K$ is stochastically complete.

For those familiar with stochastic calculus and potential theory this proposition may look almost trivial since it turns out that, in these assumptions, the set $K$ is polar with respect to the Brownian motion (i.e., it has zero capacity).

Since, by Theorem 1.9 of [13], geodesic completeness and a well-behaved sectional curvature imply the full Omori-Yau maximum principle for the Hessian, one might ask if, keeping a well-behaved sectional curvature and relaxing geodesic completeness to the property of non-extendibility, one could prove the validity of the weak maximum principle for the Hessian. This is false, as the following simple counterexample shows. Consider on Euclidean space $\mathbb{R}^{3}$ the standard cone

$$
M=\left\{x=\left(x_{1}, x_{2}, x_{3}\right) \neq(0,0,0): x_{3}=\sqrt{x_{1}^{2}+x_{2}^{2}}\right\} .
$$

In polar coordinates $(r, \theta)$, where $r \in(0,+\infty)$ and $\theta \in[0,2 \pi)$, the cone can be parametrized as $x_{1}=r \cos \theta, x_{2}=r \sin \theta, x_{3}=r$. 
Therefore, the induced metric reads

$$
d s^{2}=2 d r^{2}+r^{2} d \theta^{2}
$$

this shows that the cone is trivially non-extendible as a Riemannian manifold (every such extension $N$ must contain only one point not in $M$, but the metric is singular in $r=0$ ) but, since $M$ is a flat embedded hypersurface trivially contained into a non-degenerate cone, from Theorem 1.14 the weak maximum principle for the Hessian necessary fails. We conclude the remark observing that $M$ is stochastically complete. Indeed, from the form of the metric we deduce that the normal projection onto the hyperplane $x_{3}=0$ gives a quasi-isometry between $M$ and $\mathbb{R}^{2} \backslash\{(0,0)\}$, preserving divergent sequences and such that the derivatives of the metric on $M$ are controlled by those of $\mathbb{R}^{2} \backslash\{(0,0)\}$. Therefore, stochastic completeness follows applying a slight modification of Proposition 3.4 in [13].

Added in proof. With the article in press, we found that the very recent paper [17] is deeply related both to our work and to Atsuji's paper [1]. Indeed, the author has succeeded in recovering Atsuji's result with the aid of a geometrical approach via the weak maximum principle very similar to the one presented here and in [13].

\section{References}

[1] Atsuji, A.: Remarks on harmonic maps into a cone from a stochastically complete manifold. Proc. Japan Acad. Ser. A Math. Sci. 75 (1999), 105-108.

[2] Alías, L. J. and García-Martínez, S. C.: On the scalar curvature of constant mean curvature hypersurfaces in space forms. J. Math. Anal. Appl. 363 (2010), no. 2, 579-587.

[3] Alías, L. J., Pacelli Bessa, G. and Dajczer, M.: The mean curvature of cylindrically bounded submanifolds. Math. Ann. 345 (2009), no. 2, $367-376$.

[4] Baikoussis, C. And Kouforgiongos, T.: Harmonic maps into a cone. Arch. Math. (Basel) 40 (1983), no. 4, 372-386.

[5] Chavel, I.: Eigenvalues in Riemannian Geometry. Pure and Applied Mathematics 115. Academic Press, Orlando, FL, 1984.

[6] Chern, S. S. And Kuiper, N. H.: Some theorems on the isometric imbedding of compact Riemannian manifolds in Euclidean space. Annals of Math. (2) 56 (1952), 422-430.

[7] Dajczer, M.: Submanifolds and Isometric immersions. Mathematical Lecture Series 13. Publish or Perish, Houston, TX, 1990.

[8] Grigor'yan, A.: Analytic and geometric background of recurrence and non-explosion of the Brownian motion on Riemannian manifolds. Bull. Am. Math. Soc. 36 (1999), no. 2, 135-249. 
[9] Greene, R. And Wu, H. H.: Function theory on manifolds which possess a pole. Lecture Notes on Mathematics 699. Springer-Verlag, Berlin, 1979.

[10] Hoffman, D. And Meeks, W.: The strong halfspace theorem for minimal surfaces. Invent. Math. 101 (1990), no. 2, 373-377.

[11] Jorge, L. And Koutroufiotis, D.: An estimate for the curvature of bounded submanifolds. Amer. J. Math. 103 (1981), no. 4, 711-725.

[12] OMori, H.: Isometric immersions of Riemannian manifolds. J. Math. Soc. Japan 19 (1967), 205-214.

[13] Pigola, S., Rigoli, M. and Setti, A. G.: Maximum principles on Riemannian manifolds and applications. Memoirs of the Am. Math. Soc. $\mathbf{1 7 4}$ (2005).

[14] Pigola, S., Rigoli, M. And Setti, A. G.: A remark on the maximum principle and stochastic completeness. Proc. Amer. Math. Soc. 131 (2003), no. 4, 1283-1288.

[15] Pigola, S., Rigoli, M. and Setti, A. G.: Volume growth, "a priori" estimates and geometric applications. Geom. Funct. Anal. 13 (2003), no. 6, 1302-1328.

[16] Pigola, S., Rigoli, M. And Setti, A. G.: Maximum principles at infinity on Riemannian manifolds: an overview. Mat. Contemp. 31 (2006), 81-128.

[17] Ranjbar-Motlagh, A.: On harmonic maps from stochastically complete manifolds. Arch. Math. (Basel) 92 (2009), no. 6, 637-644.

[18] Rigoli, M., Salvatori, M. And Vignati, M.: Some remarks on the weak maximum principle. Rev. Mat. Iberoamericana 21 (2005), no. 2, 459-481.

[19] Sturm, K. T.: Analysis on local Dirichlet spaces I. Recurrence, conservativeness and $L^{p}$-Liouville properties. J. Reine Angew. Math. 456 (1994), $173-196$.

[20] Tompkins, C.: Isometric embedding of flat manifolds in Euclidean space. Duke Math. J. 5 (1939), no. 1, 58-61.

Recibido: 5 de marzo de 2009

Luciano Mari

Dipartimento di Matematica Università degli studi di Milano Via Saldini 50, I-20133 Milano (Italy)

luciano.mari@unimi.it

Marco Rigoli

Dipartimento di Matematica Università degli studi di Milano Via Saldini 50, I-20133 Milano (Italy) marco.rigoli@unimi.it 